\title{
Variação da pressão do CUFF em pacientes graves submetidos à ventilação mecânica invasiva sob os cuidados de enfermagem em unidade intensiva
}

\begin{abstract}
RESUMO | O objetivo desta pesquisa foi avaliar a variação da pressão do cuff de pacientes em unidade intensiva, no banho no leito, aspiração de vias aéreas inferiores e na mudança de decúbito, e construir um protocolo assistencial de verificação da pressão do cuff. Trata-se de um estudo descritivo, transversal, exploratório e experimental, de abordagem quantitativa, onde a coleta de dados se deu em março de 2020, em um hospital público no município de Cabo Frio. A coleta de dados foi realizada por meio de um instrumento de coleta e para verificar a pressão do cuff, foi utilizado um cufômetro artesanal, possibilitando a aferição em mmHg. Após os critérios de inclusão e exclusão, foram selecionados 10 pacientes. Ao analisarmos a variação da Pcuff durante os procedimentos realizados pela equipe de Enfermagem, a ocorrência de alterações foi frequente, sendo a mais significativa, após o banho no leito. Diante do exposto, este estudo contribui para subsidiar dados para implementação de cuidados direcionados aos pacientes intubados, a partir de ações que garantam a segurança do paciente, como a rotina de mensurações diárias e o treinamento da equipe assistencial. Protegendo assim o paciente de importantes complicações que possam estar associadas à hiperinsuflação e hipoinsuflação deste dispositivo.
\end{abstract}

Palavras-chaves: Pressão; Cuidados de Enfermagem; Cuidados Críticos; Respiração Artificial.

\begin{abstract}
The aim of this research was to evaluate the variation in cuff pressure of patients in the intensive care unit, in the bed bath, aspiration of the lower airways and in the decubitus change, and to build an assistance protocol for checking the cuff pressure. This is a descriptive, cross-sectional, exploratory, and experimental study, with a quantitative approach, where data collection took place in March 2020, in a public hospital in the municipality of Cabo Frio. Data collection was performed using a collection instrument and to check the cuff pressure, a hand-held cuffometer was used, enabling the measurement in $\mathrm{mmHg}$. After the inclusion and exclusion criteria, 10 patients were selected. When analyzing the Pcuff variation during the procedures performed by the Nursing team, the occurrence of changes was frequent, the most significant being after the bed bath. In view of the above, this study contributes to subsidize data for the implementation of care directed to intubated patients, based on actions that ensure patient safety, such as the routine of daily measurements and the training of the assistance team. Thus, protecting the patient from important complications that may be associated with the hyperinflation and hypoinflation of this device.
\end{abstract}

Keywords: Pressure; Nursing Care; Critical Care; Artificial Respiration.

RESUMEN | El objetivo de esta investigación fue evaluar la variación en la presión del manguito de los pacientes en la unidad de cuidados intensivos, en el baño de cama, la aspiración de las vías respiratorias inferiores y el cambio de decúbito, y construir un protocolo de asistencia para controlar la presión del manguito. Este es un estudio descriptivo, transversal, exploratorio y experimental, con un enfoque cuantitativo, donde la recolección de datos tuvo lugar en marzo de 2020, en un hospital público en el municipio de Cabo Frio. La recolección de datos se realizó utilizando un instrumento de recolección y para verificar la presión del manguito, se usó un cuffómetro de mano, lo que permitió la medición en mmHg. Después de los criterios de inclusión y exclusión, se seleccionaron 10 pacientes. Al analizar la variación de Pcuff durante los procedimientos realizados por el equipo de enfermería, la aparición de cambios fue frecuente, siendo la más importante después del baño en la cama. En vista de lo anterior, este estudio contribuye a subsidiar los datos para la implementación de la atención dirigida a pacientes intubados, en base a acciones que garantizan la seguridad del paciente, como la rutina de las mediciones diarias y la capacitación del equipo de asistencia. Protegiendo así al paciente de complicaciones importantes que pueden estar asociadas con la hiperinflación e hipoinflación de este dispositivo.

Palavras claves: Presión; Cuidado de Enfermera; Cuidado Crítico; Respiración Artificial.

\section{Sara de Sena Bucoski}

Enfermeira. Residente em Terapia Intensiva pela UERJ/Hospital Universitário Pedro Ernesto. RJ, Brasil.

\section{Thayná Magalhães Coutinho de Oliveira \\ Enfermeira. Graduada pela Universidade Veiga} de Almeida- Campus Cabo Frio. RJ, Brasil.

\section{Giselle Barcellos Oliveira Koeppe}

Enfermeira. Mestre e Doutora em Enfermagem. Professora do Curso de Graduação em Enfermagem da Universidade Veiga de Almeida-Campus Cabo Frio. RJ, Brasil.

\section{Priscila Pradonoff Oliveira}

Enfermeira. Mestre em Psicanálise Saúde e Sociedade. Coordenadora e Docente do Curso de Enfermagem da Universidade Veiga de Almeida Campus Cabo Frio. Especialista em Docência do Ensino Superior, Saúde Pública e Controle das Infecções Hospitalares. RJ, Brasil.

\section{Murillo Ribeiro de Mattos}

Enfermeiro. Residente em Terapia Intensiva pela UERJ/Hospital Universitário Pedro Ernesto. RJ, Brasil.

\section{Luciana da Costa Nogueira Cerqueira}

Enfermeira. Mestre em Biociência. Doutoranda em biociência da Universidade Federal do Estado do Rio de Janeiro. Docente do Curso de Graduação em Enfermagem da Universidade Veiga de Almeida-Campus Cabo Frio. RJ, Brasil.

Recebido em: 25/04/2020

Aprovado em: 26/04/2020 
INTRODUÇãO

A ventilação mecânica invasiva (VMI) tem um importante papel nas unidades intensivas, com a finalidade de promover ventilação e oxigenação adequadas quando o paciente possuir sua função ventilatória comprometida e as possibilidades terapêuticas disponíveis não apresentarem sucesso. O suporte ventilatório invasivo é realizado através da intubação traqueal, onde um tubo é inserido na traqueia por via oral ou naso, sendo este último, menos comum ${ }^{(1)}$. O tempo de permanência do tubo pode ser de curta duração, em casos de anestesia geral com finalidade cirúrgica, ou prolongado, como rotineiramente é utilizado nos pacientes internados em unidade intensiva ${ }^{(2)}$.

O tubo endotraqueal possui em sua extremidade um dispositivo denominado balonete, cuja função é verificar a pressão de ar exercida dentro do cuff( ${ }^{(2)}$. O cuff localiza-se na parte distal do tubo, sua pressão é transmitida diretamente à mucosa da traqueia, com a função de vedar a via aérea, impossibilitando o escape de ar, auxiliando na ventilação e evitando a broncoaspiração do conteúdo subglótico ${ }^{(3,4)}$. A pressão do cuff (Pcuff), deve ser avaliada com um cufômetro, este equipamento é conectado ao balonete, onde é possível visualizar os valores de pressão exercida dentro do cuff em $\mathrm{cmH} 2 \mathrm{O}$ ou $\mathrm{mmHg}$ e possibilitar o aumento ou redução da pressão, quando necessários ajustes ${ }^{(5)}$.

Em alguns estudos, reporta-se a prática de utilização da técnica de monitoramento da Pcuff por palpação manual do balonete, o que não se mostra eficaz por não corresponder à realidade da pressão ideal ${ }^{(1,6)}$.

A Pcuff ideal é descrita na literatura como de 25-30 cmH2O ou 18-22 mmHg e não deve ultrapassar a pressão de perfusão traqueal. Quando esses valores são excedidos, existe a possibilidade de gerar lesões isquêmicas da parede traqueal(5). Valores abaixo do ideal podem prejudicar a ventilação em virtude do vazamento de gás e a descida das secreções provenientes das vias aéreas superiores, mantidas em cima do balonete, predispondo à broncoaspiração. Esta última complicação é citada em estudos como sendo um fator de grande impacto para o surgimento de pneumonia associada à ventilação mecânica - PAV ${ }^{(5,7-9)}$.

Não existe consenso para a periodicidade da aferição da Pcuff, entretanto, a Diretriz Brasileira de Ventilação Mecânica sugere que seja verificada, no mínimo, quatro vezes ao dia ${ }^{(7)}$. Momento este a ser estabelecido de acordo com protocolo institucional. Porém, alterações podem ocorrer em diversos momentos, principalmente durante os cuidados de enfermagem, sendo as alterações mais significativas: o banho no leito, mudança de decúbito e aspiração de vias aéreas ${ }^{(10-12)}$.

Alguns estudos referem o distanciamento da equipe de enfermagem do processo de avaliação clínica e das ações voltadas para os pacientes com dispositivo invasivo das vias aéreas, o que pode aumentar exponencialmente os riscos de danos a esses pacientes ${ }^{(1,8)}$. Esta realidade enfatiza ainda mais a importância e relevância dos profissionais de Enfermagem como corpo científico do cuidar.

Diante deste cenário, emergiu uma questão de pesquisa: Qual comportamento da pressão do cuff nos cuidados de rotina de enfermagem?

Justifica-se o resultado do presente estudo no contexto social, relevante por contribuir para adoção de boas práticas assistenciais e, consequentemente, o aperfeiçoamento do cuidado, visando a redução de complicações pela pressão inadequada do cuff e o bem-estar do paciente assistido. Torna-se contribuinte para subsidiar e nortear a equipe de Enfermagem quanto à monitorização da pressão do cuff rotineiramente. Visto que, estudos referem que a mensuração deste dispositivo não está inserida na prática habitual de parte dos profissionais e há grande incidência de Pcuff inadequadas em pacientes sob $\mathrm{VMI}^{(2)}$. No âmbito científico, torna-se relevante para gerar dados atualizados, contribuindo para o processo de aprendizagem e elaboração de novas pesquisas acerca da temática apresentada.

\section{METODOLOGIA}

Trata-se de um estudo descritivo, transversal e experimental de abordagem quantitativa. Este estudo tem por finalidade registrar, descrever e analisar determinado fenômeno observado em uma população, sem intervir nos resultados que serão apresentados no momento ou durante um curto período. Já o estudo experimental, analisa a relação entre os acontecimentos a fim de determinar se um é a causa do outro. $\mathrm{E}$ a abordagem quantitativa, caracteriza-se por expressar as causas de um fenômeno através da linguagem matemática e a relação entre as variáveis ${ }^{(13)}$.

O presente estudo foi realizado em março de 2020 em duas unidades, em um hospital público, referência no atendimento de pacientes graves, localizado na cidade de Cabo Frio-RJ. As unidades contavam com equipe multiprofissional nas $24 \mathrm{~h}$, sendo 01 composta por 06 leitos ativos e 01 por 04 leitos, totalizando 10 leitos. Tendo como sujeitos da pesquisa 10 indivíduos.

$\mathrm{O}$ estudo atende à Resolução n. ${ }^{\circ}$ 466/2012 do Conselho Nacional de Saúde - $\mathrm{CNS}^{(14)}$, considerando os aspectos éticos e legais em pesquisas envolvendo seres humanos, sendo realizado mediante aprovação do Comitê de Ética em Pesquisa da Universidade Veiga de Almeida (CEP/UVA). Tendo o parecer consubstanciado de n. ${ }^{\circ}$ 3.961.621/2020.

A pesquisa foi realizada após o familiar, ou responsável pelo paciente, receber informações sobre a pesquisa e assinar o Termo de Consentimento Livre e Esclarecido (TCLE), visto que, os pacientes não possuíam condições clínicas de responderem por si.

Os critérios de inclusão foram pacientes submetidos à ventilação artificial por intubação traqueal e que estivessem sob ventilação mecânica invasiva. Os critérios de exclusão foram pacientes menores de 18 anos, com mudança de decúbito contraindicada, história prévia de estenose traqueal, cirurgia de cabeça e pescoço e traqueomalásia.

Os dados foram coletados no pron- 
tuário dos pacientes e registrados em um instrumento elaborado pelos autores da pesquisa, no qual continham informações sobre a identificação do paciente, idade, gênero, tempo de internação, tempo de ventilação mecânica invasiva, diagnóstico médico e pressão do cuff antes e após os procedimentos de enfermagem.

Durante a pesquisa foram analisadas as variações da pressão do cuff, após alguns procedimentos de rotina de enfermagem, sendo eles; banho no leito, mudança de decúbito e aspiração de vias aéreas inferiores. A pressão do cuff foi verificada com um aparelho artesanal, utilizando um manômetro aneróide e polifix de duas vias, possibilitando a aferição em $\mathrm{mmHg}$ quando conectado ao balonete externo do tubo endotraqueal. Esse método de verificação foi realizado por ser tratar de uma unidade que não possui um aparelho próprio para a verificação da Pcuff, o cufômetro. Os valores foram obtidos antes e após cada procedimento.

Os dados foram analisados através de tabela construída no Microsoft Excel ${ }^{\circledR}$, sendo, posteriormente, incluído em tabelas para realização de uma análise descritiva por porcentagem.

\section{RESULTADOS E DISCUSSÃO}

Durante a coleta de dados, foram encontrados 11 pacientes. Porém, um foi excluído por desistência do familiar. Sendo assim, foram selecionados 10 participantes. Após início da pesquisa, uma foi contraindicada para mudança de decúbito, tornando-se inviável a verificação da Pcuff na mudança de decúbito desta paciente. Porém, os outros procedimentos permaneceram na pesquisa.

$\mathrm{Na}$ análise sociodemográfica, pode-se identificar uma predominância da faixa etária de 60 anos ou mais (60\%). Mais representativo gênero masculino (90\%) e etnia branca $(60 \%)$, conforme apresentado na Tabela 1.

Os dados encontrados corroboram com evidências disponíveis, onde a prevalência da população idosa nas unidades de terapia intensiva está relacionada ao aumento da expectativa de vida, contribuindo para o envelhecimento populacional e, como consequência, aumento das doenças crô-

Tabela 1. Distribuição dos dados clínicos e sociodemográficos dos pacientes intubados sob ventilação mecânica invasiva em uma unidade de pacientes graves. Cabo Frio, RJ, Brasil, 2020. ( $\mathrm{N}=10)$

CARACTERÍSTICAS DEMOGRÁFICAS

$\% \mathrm{~N}$

Gênero

Masculino

$90(09)$

Feminino

$10(01)$

TOTAL

$100(10)$

Etnia

Branco

$60(06)$

Pardo

$20(02)$

Negro

$20(02)$

TOTAL

$100(10)$

Faixa Etária

$18 \vdash 40$ anos

$20(02)$

$41 \vdash 59$ anos

20 (02)

60 anos ou mais

$60(06)$

TOTAL

$100(10)$

Diagnóstico Médico

Cerebrovascular

$30(03)$

Pulmonar

$30(03)$

Cardiovascular

$20(02)$

Outros

$20(02)$

TOTAL

$100(10)$

Comorbidade Associada

Hipertensão Arterial

$80(08)$

Diabetes Mellitus

70 (07)

Sem comorbidades

$20(02)$

TOTAL

$100(10)$

\section{Tempo de Internação}

$\begin{array}{cc}1 \text { a } 10 \text { dias } & 70(07) \\ 11 \text { a } 20 \text { dias } & 20(02) \\ \text { Acima de } 20 \text { dias } & 10(01) \\ \text { TOTAL } & 100(10)\end{array}$

Tempo de Ventilação Mecânica

1 a 10 dias

$90(09)$

11 a 20 dias $0(0)$

Acima de 20 dias

$10(01)$

TOTAL $100(10)$ 
ternação em pacientes do sexo masculino, estudos demonstram que este público é mais vulnerável devido à menor adesão ao tratamento e maior exposição a riscos externos - acidentes automobilísticos e violência $^{(17,18)}$. Quanto à etnia, pode-se identificar um maior quantitativo de pessoas brancas, o que se encontra descrito em alguns estudos da mesma natureza ${ }^{(2,19)}$. Porém, vale ressaltar que a população negra é descrita na bibliográfica como uma população de grande expressão no que tange às doenças crônicas degenerativas e exposição a riscos, relacionadas a causas genéticas e ambientais, o que pode também favorecer que esse grupo venha precisar de suporte intensivo em algum período da vida ${ }^{(20)}$.

Quando analisados os dados clínicos, evidenciou-se que os motivos de internações mais frequentes foram relacionados às patologias cerebrovasculares $(30 \%)$ e pulmonares $(30 \%)$, seguidas de cardiovasculares $(20 \%)$. Tendo como principal comorbidade a Hipertensão Arterial Sistêmica (80\%). Com tempo de internação de 01 a 10 dias e tempo de ventilação mecânica de 01 a 10 dias, conforme Tabela 1.

Os dados encontrados têm similaridade com outros estudos brasileiros, onde as doenças cerebrovasculares são responsáveis por $79,6 \%$ das internações(21), além de serem a segunda maior causa de morte do mundo ${ }^{(22)}$. Esse achado também pode estar associado à média de idade dos pacientes, pois essas doenças atingem com maior frequência indivíduos acima dos $60 \operatorname{anos}^{(23,24)}$ e possuem como principal fator de risco a $\mathrm{HAS}^{(23-25)}$. No que se refere a causas pulmonares como motivo de internação, elas se apresentam com frequência nas unidades de terapia intensiva, sendo algumas delas, a pneumonia e Doença Pulmonar Obstrutiva Crônica (DPOC). Ambas são condições que afetam o trato respiratório do paciente, podendo gerar um quadro de insuficiência respiratória aguda, sendo a causa frequente de admissão na UTI e de necessidade de ventilação mecânica invasiva ${ }^{(2,26)}$. Mesmo em menor quantidade apresentada nesta pesquisa, quando comparadas às outras patologias, as doenças cardiovasculares pos- suem elevada frequência de internações em $U \mathrm{TI}^{(27,28)}$, além de serem as principais causas de morte no mundo ${ }^{(22)}$. O surgimento dessas doenças está diretamente relacionado à mudança no estilo de vida da população associado ao crescimento dos comportamentos de risco, como: dietas inadequadas, sedentarismo, tabagismo e etilismo ${ }^{(29)}$.

O tempo de internação mais encontrado girou em torno de, no máximo, 10 dias. Vale ressaltar que a permanência do paciente no ambiente de alta complexidade é um fator estressante, contribuindo para o surgimento de outras complicações, como: angustia, medo e alterações neurológicas ${ }^{(30)}$, além de contribuir na piora do prognóstico e elevar os custos de internação(31). Vale ressaltar que o perfil clínico e sociodemográfico encontrado já foi descrito por outro estudo desenvolvido na mesma unidade de saúde. Esse estudo identificou que, de 168 pacientes, a maior prevalência de internações era de homens idosos, de etnia branca e estavam relacionadas a patologias cerebrovasculares com tempo de internação menor que 10 dias $^{(19)}$. O que demostra um perfil específico da unidade. Por esse motivo, nota-se a importância de conhecer o perfil dos pacientes submetidos a suporte ventilatório invasivo, contribuindo para o aperfeiçoamento da assistência de Enfermagem e cuidado direcionado. E, consequentemente, uma melhor evolução clínica desses pacientes.

O tempo de VMI mais predominante foi de 01 a 10 dias. Já existem evidências de que o tempo de VMI está relacionado ao de internação, visto que os pacientes deste cenário costumam evoluir com necessidade de suporte ventilatório invasivo nas primeiras 24 horas da sua admissão. E quanto maior for o tempo submetido à VMI, mais suscetível se torna o paciente para o aparecimento de outras complicações. Dentre elas, podemos destacar a pneumonia associada à ventilação mecânica ${ }^{(32,33)}$. Sendo esta complicação mitigada através de medidas preventivas. Uma dessas medidas é a verificação da pressão do cuff, que necessita estar dentro dos parâmetros ideais (18 a 22 mmHg ou 25 a 30
$\mathrm{cmH} 2 \mathrm{O}$ ), a fim de evitar a broncoaspiração, se hipoinsuflado, e a lesão da mucosa traqueal, se hiperinsuflado ${ }^{(5)}$. Tendo o Enfermeiro um importante papel no que diz respeito à adesão dessas práticas por estar diretamente envolvido no cuidado, onde essas pressões podem sofrer alterações.

Dentre os cuidados de Enfermagem que podem apresentar alterações na pressão do cuff, podemos citar o banho no leito que pode sofrer variação como consequência da mobilização necessária para o banho ${ }^{(10)}$. Este procedimento é prescrito pelo Enfermeiro devido à alta complexidade que esta ação pode representar no paciente grave. Sendo assim, se faz necessário o conhecimento técnico-científico, a fim de identificar e intervir nas alterações hemodinâmicas e ventilatórias que este cuidado pode apresentar(34).

Quando analisada a variação da Pcuff nos diferentes procedimentos de enfermagem, pode-se identificar, neste estudo, que, durante o banho no leito, a elevação média foi de $11.25 \mathrm{mmHg}$ em $40 \%$ dos pacientes e a diminuição de 3.33 mmHg em 60\% dos pacientes. Na aspiração de vias aéreas inferiores houve aumento médio de $5.5 \mathrm{mmHg}$ em $20 \%$ dos pacientes e redução média 6.8 mmHg em $80 \%$ dos pacientes. Na mudança de decúbito lateral esquerdo aumentou em média 9 mmHg em 11,1\% e diminuiu 4 mmHg em 66,6\% dos pacientes, já na mudança de decúbito lateral direito, houve aumento médio de 10 mmHg em 22,2\% e diminuição de 5 mmHg em 66,6\% dos pacientes. Permaneceram com os mesmos valores pressóricos de antes do procedimento, $22,2 \%$ e $11,1 \%$ dos pacientes, respectivamente. Conforme dados da Tabela 2.

Becarria $^{(9)}$ aponta a importância da verificação da Pcuff após os cuidados de enfermagem, principalmente após o banho no leito. Visto que esse procedimento apresentou redução da pressão intra-cuff na maior parte dos pacientes intubados. Podendo gerar como consequências, a pneumonia associada a ventilação mecânica, o escape de ar prejudicando a ventilação, entre ou$\operatorname{tros}^{(3-5,9)}$. Em relação ao aumento da pressão após o banho no leito, este fator pode estar 
Tabela 2. Variação da pressão do Cuff de pacientes submetidos a ventilação mecânica invasiva sob os cuidados de Enfermagem na Unidade de pacientes graves. Cabo Frio, RJ, Brasil, 2020.

\begin{tabular}{|c|c|c|}
\hline Procedimentos de Enfermagem & $\begin{array}{c}\text { Após procedimento } \\
\% \mathrm{~N}\end{array}$ & $\begin{array}{l}\text { Média da variação da } \\
\text { pcuff } \mathrm{mmHg}\end{array}$ \\
\hline \multicolumn{3}{|l|}{ Banho no leito } \\
\hline Aumentou & 40 (4) & 11.25 \\
\hline Diminuiu & 60 (6) & 3.33 \\
\hline Permaneceu & $0(0)$ & \\
\hline TOTAL & $100(10)$ & \\
\hline \multicolumn{3}{|l|}{ Aspiração de via aérea inferior } \\
\hline Aumentou & $20(2)$ & 5.5 \\
\hline Diminuiu & $80(8)$ & 6.8 \\
\hline Permaneceu & $0(0)$ & \\
\hline TOTAL & $100(10)$ & \\
\hline \multicolumn{3}{|l|}{ Decúbito lateral esquerdo } \\
\hline Aumentou & $11,1(1)$ & 9 \\
\hline Diminuiu & $66,6(6)$ & 4 \\
\hline Permaneceu & $22,2(2)$ & \\
\hline TOTAL & $100(9)$ & \\
\hline \multicolumn{3}{|l|}{ Decúbito lateral direito } \\
\hline Aumentou & $22,2(2)$ & 10 \\
\hline Diminuiu & $66,6(6)$ & 5 \\
\hline Permaneceu & $11,1(1)$ & \\
\hline TOTAL & $100(9)$ & \\
\hline
\end{tabular}

associado em decorrência da compressão do tubo sobre o cuff causada pela movimentação ${ }^{(11)}$. A elevação desta pressão sob a mucosa traqueal, acima dos valores de referência, pode gerar complicações, tais como: estenose, laringite, ulceração da mucosa, isquemia, hemorragia, entre ou$\operatorname{tros}^{(1,2,5,9)}$. Tais complicações podem dificultar a extubação do paciente, favorecendo o aumento no tempo de hospitalização e, consequentemente, acréscimo dos custos de internação ${ }^{(31)}$.

Outro cuidado de enfermagem que provoca alterações na Pcuff é a aspiração de vias aéreas inferiores ${ }^{(10)}$. A aspiração é realizada com objetivo de remover as secreções acumuladas, favorecendo a permeabilidade das vias aéreas e, consequentemente, melhorando a ventilação e oxigenação. Já que pacientes em VMI tendem ao acúmulo de secreções respiratórias devido à tosse ineficaz e à presença do tubo prejudicando o fechamento da glote; o acúmulo das secreções elevam as chances de desenvolver PAV em consequência da broncoaspiração( ${ }^{(3)}$. Ressaltando o importante papel do Enfermeiro frente à esta ação, a fim de prevenir PAV pelo acúmulo de secreções ou pela diminuição que a pressão do cuff pode apresentar após a aspiração. Os dados encontrados no presente estudo corroboram com outra pesquisa, que demonstrou diminuição da pressão do cuff após a aspiração de secreções endotraqueais ${ }^{(10)}$. No que diz respeito ao aumento da Pcuff em alguns pacientes, após a aspiração de vias aéreas inferiores, este achado pode estar relacionado a marca do tubo utilizado ou na rotina de procedimentos realizados por outros profissionais de saúde. O que não foi uma variável investigada pelo presente estudo.

Em relação à mudança de decúbito, sa- be-se que esta atividade tem como objetivo prevenir Lesões por Pressão (LP) e complicações cardiopulmonares. Sendo este um procedimento complexo, que requer do enfermeiro conhecimento técnico-científico, com finalidade de identificar alterações hemodinâmicas e ventilatórias que esta ação pode causar ${ }^{(36,37)}$. Dentre as alterações motivadas pela mudança de decúbito, podemos citar a variação da Pcuff ${ }^{(2,10,11)}$. Um estudo semelhante identificou uma média de elevação da Pcuff de 25 para $32,59 \mathrm{cmH} 2 \mathrm{O}$, o que equivale a uma variação de 18 para $24 \mathrm{mmHg}$ em $47,3 \%$ dos pacientes estudados, após a mudança de decúbito(11), o que corrobora com os resultados encontrados nesta pesquisa, visto que, parte dos pacientes teve a pressão do cuff aumentada após a mudança de decúbito. Uma outra pesquisa, identificou que $58,7 \%$ dos pacientes intubados apresentaram redução da Pcuff após mudança de decúbito ${ }^{(12)}$, corroborando com os dados encontrados no presente estudo, onde a maioria dos pacientes também tiveram a Pcuff diminuída após a mudança de decúbito.

\section{CONCLUSÃO}

Conclui-se que a maioria dos sujeitos da pesquisa eram homens brancos e idosos, com prevalência dos diagnósticos cerebrovasculares e pulmonares. Tendo a hipertensão arterial sistêmica como a maior comorbidade associada. E tempo de internação e ventilação mecânica entre 1 a 10 dias.

O presente estudo teve como objetivo analisar a variação da pressão do cuff dos pacientes intubados sob ventilação mecânica invasiva no banho no leito, mudança de decúbito, aspiração de vias aéreas inferiores e construir um formulário de acompanhamento da Pcuff

Em todos os procedimentos de Enfermagem houve prevalência na diminuição da pressão do cuff, mas também existiu um número expressivo de elevações, principalmente após o banho no leito. O que corrobora com alguns estudos, conforme já citados neste trabalho. Demonstrando que não existe concordância entre a elevação 
e diminuição da Pcuff na bibliografia. Por isso, destaca-se a importância de entender, em cada unidade de saúde, o perfil de variação deste dispositivo no paciente, para que seja prestada uma assistência de Enfermagem qualificada e direcionada, através da implementação de medidas para prevenção de lesões traqueais e broncoaspiração.

A limitação do estudo ficou restrita ao tempo de realização e complexidade de análise e a inclusão dos pacientes, o que dificultou algumas discussões associadas.
Ressalta-se que houve um número de pacientes analisados, relativamente pequeno, associado à complexidade da concretização de estudos experimentais. Porém, pesquisas semelhantes apresentaram número similar de pacientes estudados ${ }^{(2,11)}$.

\section{Referências}

1. Pinto DM, Schons ES, Busanello J, Costa VZ. Segurança do paciente e a prevenção de lesões cutâneo-mucosas associadas aos dispositivos invasivos nas vias aéreas. Rev Esc Enfermagem USP. 2015 out; 49(5): 775-782. 2. Barroso AKS, Soares JS. Análise da pressão do cuff/balonete em pacientes sob ventilação mecânica invasiva na unidade de terapia intensiva (UTI). Brazilian Journal of Surgery and Clinical Research. 2017 nov; 20(1): 07-20. 3. Hinkle JL, Cheever KH. Kerry H. Brunner \& Suddarth tratado de enfermagem médico-cirúrgica. 13. ed. Rio de Janeiro-RJ: Guanabara Koogan; 2016. 4. Mogal SS, Baliarsing L, Dias R, Gujjar P. Comparação de alterações na pressão do balonete do tubo endotraqueal usando ar versus óxido nitroso nos gases anestésicos durante cirurgias abdominais laparoscópicas. Rev Bras Anestesiol. 2018 jan; 68(4): 369-374.

5. Agência Nacional de Vigilância Sanitária em Saúde (BR). Medidas de prevenção de infeccão relacionada à assistencia à saúde. Brasília (DF): ANVISA, 2017. 6. Giusi GD, Rogari C, Gili A, Nisi F. Cuff pressure monitoring by manual palpation in intubated patients: How accurate is it? A manikin simulation study. Australia Critical Care. 2017 jul; 30(4): 234-8.

7. Medicina Intensiva Brasileira. Diretrizes Brasileiras de Ventilação Mecânica. 2013:1-136

8. Maran E, Spigolon DN, Melo WA, Barreto MS, Tostes MFP, Teston EF. Prevenção da pneumonia associada à ventilação mecânica sob a ótica de acadêmicos de enfermagem. Cuidado é fundamental. 2019 mar; 11(1): 118-123. 9. Cruz JRM, Martins MDS. Pneumonia associada à ventilação mecânica invasiva: cuidados de enfermagem. Rev Enf Ref. 2019 mar; IV (20): 87-96. 10. Becarria LM, Doimo TMA, Polletti NAA, Barbosa TP, Silva DC, Werneck AL. Mudança de pressão do balonete traqueal antes e depois da realização dos cuidados de enfermagem. Rev. Bras. Enferm. 2017 dez; 70(6): 1145-1150.

11. Alcan AO, Giersbergen MYV, Dincarslan G, Hepcivici Z, Kaya E, Uyar M. Effect of patient position on endotracheal cuff pressure in mechanically ventilated critically ill patients. Australian Critical Care. 2017 set; 30(5): 267-272. 12. Ferreira EG, Rickli P, Molina Filho ET, Fischer J, Antunes MD, Nascimento Junior JRA, Oliveira DV. Pressão do cuff em pacientes em ventilação mecânica-relação com a mudança de decúbito. Ciência e Saúde. 2017; 10(2): 114-119. 13. Hulley BS, Cummings RS, Browner SW, Grady GD Newman BT. Delineando a pesquisa Clínica. 4. Ed. Porto Alegre (RS): Artmed; 2015.

14. Conselho Nacional de Saúde (BR). Resolução n. ${ }^{\circ} 466$, de 12 de dezembro de 2012. Diretrizes e normas regulamentadoras de pesquisas envolvendo seres humanos. Diário Oficial da União 13 Jun 2013; Seção 1.

15. Organização Mundial da Saúde, Organização Pan-Americana da Saúde. Folha informativa - Envelhecimento e saúde. Brasília (DF): OMS/OPAS, 2018. 16. Rodriguez AH, Bub MBC, Perao OF, Zandonadi G, Rodriguez MJH. Características epidemiológicas e causas de óbitos em pacientes internados e terapia intensiva. Rev Bras Enferm. 2016 set; 69(2): 210-4.

17. Castro RR, Barbosa NB, Alves T, Najberg E. Perfil das internações em unidade de terapia intensiva adulto na cidade de Anápolis - Goiás em 2012. Revista de Gestão em Sistemas de Saúde. 2016 mar; 5(2).

18. Perão OF, Bub MBC, Zandonadi GC, Martins MA. Características sociodemográficas e epidemiológicas de pacientes internados em uma unidade de terapia intensiva de adultos. Rev Enferm UERJ. 2017 mar; 25:7736.

19. Pinto DS, Silva BAA, Koeppe GBO, Pereira SP, Teixeira PC, Cerqueira LCN. Descrição clínica e sociodemográfica de pacientes internados em uma Unidade de Pacientes Graves de Cabo Frio-RJ. Revista Nursing. 2019; 22 (259): 3431-35. 20. Ministério da Saúde (BR). Painel de indicadores do SUS nº 10. Temático
Saúde da População negra. v.VII. Brasília (DF): MS, 2016.

21. Melo EM, Barbosa AA, Silva JLA, Sombra RLS, Studart RMB, Lima FET, et al. Evolução Clínica dos Pacientes em uso de ventilação mecânica em unidade de terapia intensiva. Rev Enferm UFPE. 2015 fev; 9(2): 610-1.

22. Organização Mundial da Saúde, Organização Pan-Americana da Saúde. 10 principais causas mortes do mundo. Brasília (DF): OMS/OPAS, 2018.

23. Lotufo PA, Goulart AC, Passos VMA, Satake FM, Souza MFM, França EB, et al. Doença cerebrovascular no Brasil de 1990 a 2015: Global Burden of Disease 2015. Rev. bras. epidemiol. 2017 mai; 20(1):129-141.

24. Oliveira JG, Damasceno KG, Souza LP, Lima MG. Perfil clínico epidemiológico e os principais rótulos diagnósticos de enfermagem aos pacientes internados com acidente vascular cerebral em um hospital de grande porte na região sul da Amazônia legal. Revista Amazônia Science \& Health. 2016 set; 4(3):3-11.

25. Melo EM, Oliveira TMM, Marques AM, Ferreira AMM, Silveira FMM, Lima VF. Caracterização dos pacientes em uso de drogas vasoativas internados em unidade de terapia intensiva. Cuidado é fundamental. 2016 set; 8(3):129-141 26. França CDM, Albuquerque PR, Santos ACBC. Perfil epidemiológico da unidade de terapia intensiva de um Hospital Universitário. Revista InterScientia. 2016 dez; 1(2):72-82

27. Perão OF, Bub MBC, Zandonadi GC, Martins MA. Características sociodemográficas e epidemiológicas de pacientes internados em uma unidade de terapia intensiva de adultos. Rev enferm UERJ. 2017 mar; 25:e7736.

28. Organização Mundial da Saúde, Organização Pan-Americana da Saúde. Doenças cardiovasculares. Brasília (DF): OMS/OPAS, 2017.

29. Organização Mundial da Saúde, Organização Pan-Americana da Saúde. Doenças crônicas não transmissíveis causam 16 milhões de mortes prematuras todos os anos. Brasília (DF): OMS/OPAS, 2015.

30. Membrive AS, Souza LPS, Donoso MTV, Silqueira SMF, Corrêa AR, Matos SS. Caracterização dos estressores envolvidos na internação de pacientes em unidade coronariana. Rev baiana de Enfermagem. 2017; 31(1):e16552. 31. Sinésio MCT, Magro MCS, Carneiro TA, Silva KGN. Fatores de risco às infecções relacionadas à assistência em unidades de terapia intensiva. Cogitare Enferm. 2018; 23(2):e53826.

32. Freitas MA, Aragones BL, Fleck CS. Perfil clínico de pacientes submetidos à ventilação mecânica em uma unidade de pronto atendimento da região central do Rio Grande do Sul. Fisioterapia Brasil. 2019 jun; 20 (4): 476-484. 33. Frota ML, Campanharo CRV, Lopes MCBT, Piacezzi LHV, Okuno MFP, Batista REA. Boas práticas para prevenção de pneumonia associada à ventilação mecânica no serviço de emergência. Revista da Escola de Enfermagem da USP. 2019 jun; 53:e0460.

34. Bastos SRB, Gonçalves FAF, Bueno BRM, Silva GS, Ribeiro KRA, Brasil VV. Banho no Leito: Cuidados Omitidos pela Equipe de Enfermagem. J res fundam care. 2019 jun; 11(3): 627-63.

35. Lopes VJ, Muller F, Souza MAR, Silva IA. Aspiração endotraqueal em pacientes com via aérea artificial sob ventilação mecânica invasiva internados em UTI. Revista de Enfermagem do Centro-Oeste Mineiro. 2018; 8:e1973. 36. Magnus LM, Backes MTS, Backes DS. Mudança de decúbito em pacientes com injúria cerebral grave: construção de um guia com enfermeiros intensivistas. Enferm. Foco. 2018; 9(2):28-34.

37. Oliveira TMC, Bucoski SS, Koeppe GBO, Santos AG, Pereira LS, Cerqueira LCN. Repercussões hemodinâmicas e ventilatórias do paciente em ventilação mecânica invasiva na mudança de decúbito. Revista Nursing. 2020 23(261):3600-06. 